\title{
On the numerical simulation of material inhomogeneities due to martensitic phase transformations in poly-crystals
}

\author{
Philipp Junker ${ }^{1, a}$ and Klaus Hackl ${ }^{1}$ \\ Institut für Mechanik, Ruhr-Universität Bochum, Universitätsstraße 150, 44801 Bochum, Germany
}

\begin{abstract}
Experimental results show that martensitic phase transformations in shape-memory alloys occur in localized zones. Based on a micromechanical model we want to simulate these effects. The mathematical treatment is accompanied by a finite-element calculation which includes, due to the model modification, a regularization. The result of this effort is a micromechanically well motivated algorithm which shows qualitatively a good agreement with experiments. Numerical examples are presented.
\end{abstract}

\section{Introduction}

Shape-memory alloys are materials with high technological interest due to their ability of solidto-solid phase transformations. These transformations give rise to the so-called effects of pseudoelasticity (at temperatures above the transformation temperature) or pseudo-plasticity (at temperatures below the transformation temperature). Thus specimens made of shape-memory alloys have a large variety of applications, such as stents, actors or thin film foils.

Since the understanding of so-called smart materials can only proceed using both experimental and theoretical approaches, this paper intends to contribute to the theoretical part of this research field. Using an elastic energy derived in [7], we are able to formulate the equilibrium conditions for continuous bodies by energy minimization. These equilibrium conditions can be solved by a finite-element approach so that the material behavior such as force-displacement relations and phase distributions can be calculated for entire specimens made of shape-memory alloys. For this approach, however, results do not coincide with experimental findings because they do not reflect the inhomogeneous material response. Hence, we present a modified model which is supposed to yield to more realistic results, but which has to be treated in a different mathematical way.

Regarding the high number of existing models for shape-memory alloys, we can only mention a small number of them. Phenomenological models were presented in [2] and [9], while [1], [16], [10] and [18] established microscopic models.

The modeling of the multi-axial behavior of shape-memory alloys by a finite-element implementation can be found in [14] where a model introduced in [17] is used.

The micromechanical model for polycrystalline shape-memory alloys used in this paper was presented by [7]. This model is based on a combination of works in [4], [5], [7] and [8].

First we recall in Section 2 the used micromechanical model and develop our new attempt. In Section 2.2 and 3 we discuss the resulting mathematical statement of the problem for which we present a solution strategy. At the end we show numerical results for a specific boundary value problem.

\footnotetext{
${ }^{a}$ e-mail: philipp.junker@rub.de
} 


\section{Micromechanical model}

\subsection{Relaxed energy}

The micromechanical model is applied to poly-crystalline materials. Each grain in such a polycrystal is supposed to possess a certain orientation which is identified by a certain rotation of the global coordinate system. We consider a representative volume of the poly-crystal to contain $N$ crystallites where each one is denoted by a number $j$. Since recrystallization is excluded, the number of grains and the relative volume fraction of each grain do not change during calculation. The rotation matrices are distributed randomly.

The two main phases, austenite and martensite, have different elastic constants which are collected separately in the corresponding stiffness tensors $\mathbb{C}_{i}$. The index $i$ refers to the specific phase ( $i=0$ means the austenitic phase, $i \neq 0$ means the corresponding martensitic phase). The elastic constants are the same for all different martensite variants. Since the stiffness tensor depends on the corresponding coordinate system, $\mathbb{C}$ has to be rotated into the direction of each grain utilizing the rotation matrices. Therefore we have for each variant $i$ in each direction $j$ a specific stiffness tensor $\mathbb{C}_{i}^{j}$.

The different martensitic variants can be distinguished by their different transformation strains $\boldsymbol{\eta}_{i}$ which are know from experiments. These transformation strains have once again to be rotated into the direction of each crystallite which leads to $\boldsymbol{\eta}_{i}^{j}$.

Assuming linear elastic material behavior for each phase in each grain, the energy can be written as

$$
\Psi\left(\varepsilon_{i}^{j}\right)=\frac{1}{2}\left(\varepsilon_{i}^{j}-\boldsymbol{\eta}_{i}^{j}\right): \mathbb{C}_{i}^{j}:\left(\varepsilon_{i}^{j}-\boldsymbol{\eta}_{i}^{j}\right)+\alpha_{i},
$$

while $\alpha_{i}$ is the chemical energy which controls whether the austenite is stable at zero strain or not. Hence we can write $\alpha_{0} \neq 0, \alpha_{i}=0 \forall i \neq 0$.

The amount of each phase within each grain is arranged in a matrix $\boldsymbol{\lambda}$. So the component $\lambda_{i}^{j}$ gives the volume fraction of phase $i$ in the crystallite $j$. We have to impose mass conservation

$$
\sum_{i=0}^{n} \lambda_{i}^{j}=1 \quad \forall \quad j=1, \ldots, N,
$$

and that the average of the strains of all phases in all directions has to yield the macroscopic strain $\varepsilon$

$$
\varepsilon=\sum_{j=1}^{N} \sum_{i=0}^{n} \xi^{j} \lambda_{i}^{j} \varepsilon_{i}^{j}
$$

Under the constraints (2) and (3) the total energy of the system has to be found for fixed volume fractions $\boldsymbol{\lambda}$. Consequently, an approximation by convexification of the relaxed energy of the whole body reads

$$
\Psi^{\mathrm{rel}}(\boldsymbol{\varepsilon}, \boldsymbol{\lambda})=\inf _{\boldsymbol{\varepsilon}_{i}^{j}}\left\{\sum_{j=1}^{N} \sum_{i=0}^{n} \xi^{j} \lambda_{i}^{j} \Psi_{i}^{j}\left(\varepsilon_{i}^{j}\right) \mid \varepsilon=\sum_{j=1}^{N} \sum_{i=0}^{n} \xi^{j} \lambda_{i}^{j} \varepsilon_{i}^{j}\right\},
$$

and minimization gives, [7]

$$
\Psi^{\mathrm{rel}}(\boldsymbol{\varepsilon}, \boldsymbol{\lambda})=\frac{1}{2}\left(\boldsymbol{\varepsilon}-\boldsymbol{\eta}_{\mathrm{eff}}\right): \mathbb{C}_{\mathrm{eff}}:\left(\boldsymbol{\varepsilon}-\boldsymbol{\eta}_{\mathrm{eff}}\right)+\alpha_{\mathrm{eff}},
$$

with the effective variables

$$
\mathbb{C}_{\mathrm{eff}}=\left[\sum_{j=1}^{N} \sum_{i=0}^{n} \xi^{j} \lambda_{i}^{j}\left(\mathbb{C}_{i}^{j}\right)^{-1}\right]^{-1}, \quad \boldsymbol{\eta}_{\mathrm{eff}}=\sum_{j=1}^{N} \sum_{i=0}^{n} \xi^{j} \lambda_{i}^{j} \boldsymbol{\eta}_{i}^{j}, \quad \alpha_{\mathrm{eff}}=\sum_{j=1}^{N} \sum_{i=0}^{n} \xi^{j} \lambda_{i}^{j} \alpha_{i} .
$$




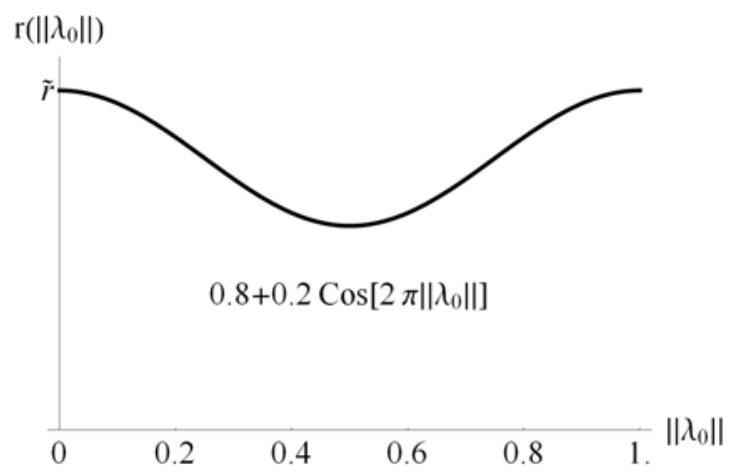

Fig. 1. Assumed dependency between the dissipation and the total amount of austenite $\left(r\left(\left\|\boldsymbol{\lambda}_{0}\right\|\right)\right)$.

The components $\xi^{j}$ describe the volume fraction of each orientation $j$. Here all orientations are assumed to have the same probability, hence $\xi^{j}=1 / N \forall j$.

\subsection{Evolution equations}

Since maximization of the entropy equals minimization of the total power [6], we minimize a Lagrange functional which has the form of the total power. In the micromechanical model in [7], a Lagrange functional of the form

$$
\tilde{\mathcal{L}}(\boldsymbol{\lambda}, \dot{\boldsymbol{\lambda}})=\frac{\mathrm{d}}{\mathrm{d} t} \Psi^{\mathrm{rel}}(\varepsilon, \boldsymbol{\lambda})+\Delta(\dot{\boldsymbol{\lambda}})
$$

was used, in which $\Delta(\dot{\boldsymbol{\lambda}})$ is the ansatz for the entropy production. Since this approach yields experimentally not observed homogeneous transformations, we modify this ansatz by setting

$$
\Delta\left(\left\|\boldsymbol{\lambda}_{0}\right\|, \dot{\boldsymbol{\lambda}}\right)=r\left(\left\|\boldsymbol{\lambda}_{0}\right\|\right) \sqrt{\sum_{j=1}^{N} \sum_{i=0}^{n} \xi^{j}\left(\dot{\lambda}_{i}^{j}\right)^{2}},
$$

where $r\left(\left\|\boldsymbol{\lambda}_{0}\right\|\right)=$ const was used in [7] and in our case the dissipation coefficient $r$ is assumed to be a function of the total amount of austenite

$$
\left\|\boldsymbol{\lambda}_{0}\right\|=\sum_{j=1}^{N} \xi^{j} \lambda_{0}^{j} .
$$

Starting from a pure austenite state (pseudo-elasticity), the evolution of the martensitic phases is assumed to dissipate less energy than a nucleation of martensite within the pure austenite state. At a later point of evolution, the complete disappearance of austenite costs more energy than an interchange between the already existing martensitic phases (a constant amount of austenite is preferred). These assumptions have to be taken into account by making an ansatz for the function $r\left(\left\|\boldsymbol{\lambda}_{0}\right\|\right)$.

As function $r\left(\left\|\boldsymbol{\lambda}_{0}\right\|\right)$ for the dissipation, we assume a cos-function which is shown in Figure (1).

Minimization of the introduced Lagrange functional $\tilde{\mathcal{L}}$ with respect to the volume fractions $\boldsymbol{\lambda}$ gives the evolution equations. A straight forward strategy to pursue this approach, however, would lead to mesh-dependent strain localizations. To avoid this, we use a method introduced in [3]. Mesh-depending localizations occur as a result of lacking convexity of our energy. This can be remedied by introducing convex terms in higher order gradients of the corresponding variables.

This extension, however, would lead to a "global" yield function which determines whether the transformation takes place in the whole body or not. 
Since the transformation depends on the location within the body, local yield functions are desired. Hence, not the gradient of the internal variables itself is used to expand the energy, but a "nonlocal" field $\varphi$ is introduced which is supposed to transport the informations on the internal variables throughout the body. Therefore the energy is extended by the gradient of this field and by a second part which penalizes the discrepancy between it and the (local) internal variable. This yields as energy (for super-elasticity)

$$
\Psi\left(\varepsilon, \lambda,\left\|\lambda_{0}\right\|, \varphi\right)=\Psi^{\mathrm{rel}}(\varepsilon, \boldsymbol{\lambda})+\frac{c_{\varphi}}{2}\|\nabla \varphi\|^{2}+\frac{\beta_{\varphi}}{2}\left(\varphi+1-\left\|\boldsymbol{\lambda}_{0}\right\|\right)^{2} .
$$

Substituting this energy into the Lagrange functional gives

$$
\begin{aligned}
\mathcal{L}(\boldsymbol{\lambda}, \dot{\boldsymbol{\lambda}}) & =\frac{\mathrm{d}}{\mathrm{d} t} \Psi\left(\varepsilon, \boldsymbol{\lambda},\left\|\boldsymbol{\lambda}_{0}\right\|, \varphi\right)+\Delta\left(\left\|\boldsymbol{\lambda}_{0}\right\|, \dot{\boldsymbol{\lambda}}\right) \\
& =\frac{\mathrm{d}}{\mathrm{d} \boldsymbol{\lambda}} \Psi\left(\varepsilon, \boldsymbol{\lambda},\left\|\boldsymbol{\lambda}_{0}\right\|, \varphi\right): \frac{\mathrm{d} \boldsymbol{\lambda}}{\mathrm{d} t}+\Delta\left(\left\|\boldsymbol{\lambda}_{0}\right\|, \dot{\boldsymbol{\lambda}}\right)
\end{aligned}
$$

The conjugated driving forces can be identified as

$$
\begin{aligned}
& q_{i}^{j}=-\frac{\mathrm{d}}{\mathrm{d} \lambda_{i}^{j}} \Psi\left(\varepsilon, \boldsymbol{\lambda},\left\|\boldsymbol{\lambda}_{0}\right\|, \varphi\right) \\
&=\xi^{j}\left[\boldsymbol{\eta}_{i}^{j}: \mathbb{C}_{\mathrm{eff}}:\left(\boldsymbol{\varepsilon}-\boldsymbol{\eta}_{\mathrm{eff}}\right)+\frac{1}{2}\left(\varepsilon-\boldsymbol{\eta}_{\mathrm{eff}}\right):\left(\mathbb{C}_{\mathrm{eff}}:\left(\mathbb{C}_{i}^{j}\right)^{-1}: \mathbb{C}_{\mathrm{eff}}\right):\left(\boldsymbol{\varepsilon}-\boldsymbol{\eta}_{\mathrm{eff}}\right)-\alpha_{i}\right] \\
& \quad+\underbrace{\beta_{\varphi} \xi^{j}\left(\varphi+1-\left\|\boldsymbol{\lambda}_{0}\right\|\right) \delta_{0 i}}_{\star} .
\end{aligned}
$$

The last part $(\star)$ in equation $(12)$ connects the evolution of the internal variables with the nonlocal field. $\delta_{0 i}$ is the Kronecker delta; hence just the evolution of the austenite phase is influenced directly by $\varphi$.

In an analogous way to [7] the evolution equations for the different phases can be derived as

$$
\begin{aligned}
& q_{i}^{j}-\frac{1}{n_{\mathcal{A}^{j}}} \sum_{k \in \mathcal{A}^{j}} q_{k}^{j}=r\left(\left\|\boldsymbol{\lambda}_{0}\right\|\right) \xi^{j} \frac{\dot{\lambda}_{i}^{j}}{\sqrt{\sum_{j=1}^{N} \sum_{i=0}^{n}\left(\dot{\lambda}_{i}^{j}\right)^{2}}} \text { for } i \in \mathcal{A}^{j} \\
& \left(\sum_{j=1}^{N} \sum_{i=0}^{n} \frac{1}{\xi^{j}}\left(q_{i}^{j}-\frac{1}{n_{\mathcal{A}^{j}}} \sum_{k \in \mathcal{A}^{j}} q_{k}^{j}\right)^{2}\right)^{1 / 2} \leq r\left(\left\|\boldsymbol{\lambda}_{0}\right\|\right) \quad \text { for } \quad i \in \mathcal{A}^{j} \quad \text { and } \quad \dot{\lambda}_{i}^{j}=0 \\
& q_{i}^{j}-\frac{1}{n_{\mathcal{A}^{j}}} \sum_{k \in \mathcal{A}^{j}} q_{k}^{j}<0 \text { for } i \notin \mathcal{A}^{j},
\end{aligned}
$$

by introducing the passive set $\mathcal{B}^{j}$ and the active set $\mathcal{A}^{j}$ in each grain as

$$
\begin{aligned}
& \mathcal{B}^{j}=\left\{i \mid \lambda_{i}^{j}=0\right\}, \\
& \mathcal{A}^{j}=\left\{i \in \mathcal{B}^{j} \mid \dot{\lambda}_{i}^{j}>0\right\} \cup\left\{i \notin \mathcal{B}^{j}\right\}
\end{aligned}
$$

and $n_{\mathcal{A}^{j}}$ is the number of active variants in each phase. Equation (13) describes the evolution of the active phases and equation (15) serves as a switch between the active and the passive 
sets. The left part of both equations is called active deviator and denoted by $\operatorname{dev}_{\mathcal{A}^{j}} q_{i}^{j}=q_{i}^{j}-$ $\frac{1}{n_{\mathcal{A}^{j}}} \sum_{k \in \mathcal{A}^{j}} q_{k}^{j}$.

To simplify the numerical implementation, a Legendre transformation of the Lagrange functional $\mathcal{L}$ is introduced as

$$
\begin{aligned}
\mathcal{J}(\boldsymbol{q}) & =\sup \left\{\left(\operatorname{dev}_{\mathcal{A}} \boldsymbol{q}\right) \dot{\boldsymbol{\lambda}}-\Delta \mid \dot{\boldsymbol{\lambda}}\right\} \\
& =\sup \{\Delta \underbrace{\left(\sum_{j=1}^{N} \frac{1}{\xi^{j}} \sum_{i=0}^{n}\left(\operatorname{dev}_{\mathcal{A}^{j}} q_{i}^{j}\right)^{2}-\left(r\left(\left\|\boldsymbol{\lambda}_{0}\right\|\right)\right)^{2}\right)}_{=: \Phi(\boldsymbol{q})}\}
\end{aligned}
$$

which gives a yield function $\Phi(\boldsymbol{q})$ similar to the yield functions known from plasticity so that

$$
\mathcal{J}(\boldsymbol{q})=\left\{\begin{array}{cc}
0 & \text { for } \quad \Phi(\boldsymbol{q}) \leq 0 \\
\infty & \text { else }
\end{array} .\right.
$$

Then equation (13) gives

$$
\dot{\lambda}_{i}^{j}=\frac{\rho}{\xi^{j}}\left[\operatorname{dev}_{\mathcal{A}^{j}} q_{i}^{j}\right]_{\mathcal{A}^{j}},
$$

with the Kuhn-Tucker conditions

$$
\rho:=\frac{\Delta}{\left(r\left(\left\|\boldsymbol{\lambda}_{0}\right\|\right)\right)^{2}} \geq 0, \quad \Phi \leq 0, \quad \rho \Phi=0
$$

and the consistency condition

$$
\operatorname{dev}_{\mathcal{A}^{j}} q_{i}^{j} \leq 0 \quad \text { for } \quad i \notin \mathcal{A}^{j} .
$$

\section{Finite-element implementation}

\subsection{Finite-element approach}

For the finite-element implementation the potential function $\Pi$ is used as

$$
\Pi=\int_{\Omega} \Psi \mathrm{d} V-\int_{\Omega} \boldsymbol{u} \cdot(\rho \boldsymbol{b}) \mathrm{d} V-\int_{\partial \Omega} \boldsymbol{u} \cdot \boldsymbol{t} \mathrm{d} A,
$$

where we have $\rho \boldsymbol{b}$ as body force per unit volume of the body and $\boldsymbol{t}$ as some external force on the boundary of the body $\partial \Omega$. This potential depends directly on the displacements $\boldsymbol{u}$ and on the nonlocal field $\varphi$. In order to minimize this energy we find the variation of the potential with respect to $\boldsymbol{u}$ and $\varphi$ and set these equations to zero. That gives

$$
\begin{array}{ccc}
\int_{\Omega} \delta \varepsilon: \frac{\partial \Psi}{\partial \boldsymbol{\varepsilon}} \mathrm{d} V-\int_{\Omega} \delta \boldsymbol{u} \cdot(\rho \boldsymbol{b}) \mathrm{d} V-\int_{\partial \Omega} \delta \boldsymbol{u} \cdot \boldsymbol{t} \mathrm{d} A=0 & \forall \delta \boldsymbol{u} \\
\int_{\Omega} c_{\varphi}(\nabla \varphi \cdot \nabla \delta \varphi) \mathrm{d} V+\int_{\Omega} \beta_{\varphi}\left(\varphi+1-\left\|\boldsymbol{\lambda}_{0}\right\|\right) \delta \varphi \mathrm{d} V=0 & \forall \delta \varphi
\end{array}
$$

These two equations can now be solved by a finite-element approach in a standard manner. We use shape functions called $\boldsymbol{N}_{\boldsymbol{u}}$ and $\boldsymbol{N}_{\varphi}$ to approximate the exact functions $\boldsymbol{u}$ and $\varphi$ respectively as 


$$
\boldsymbol{u}=\boldsymbol{N}_{\boldsymbol{u}} \cdot \hat{\boldsymbol{u}}, \quad \boldsymbol{\varphi}=\boldsymbol{N}_{\varphi} \cdot \hat{\boldsymbol{\varphi}}
$$

with $\hat{\boldsymbol{u}}$ and $\hat{\boldsymbol{\varphi}}$ as vectors of the nodal values of $\boldsymbol{u}$ and $\varphi$. Since $\boldsymbol{u}$ is a vectorial and $\varphi$ a scalar quantity, $\boldsymbol{N}_{\boldsymbol{u}}$ is a matrix and $\boldsymbol{N}_{\varphi}$ a vector respectively. Using (26) we obtain

$$
\begin{array}{cl}
\delta \boldsymbol{u}=\boldsymbol{N}_{\boldsymbol{u}} \cdot \delta \hat{\boldsymbol{u}}, & \delta \varphi=\boldsymbol{N}_{\varphi} \cdot \delta \hat{\boldsymbol{\varphi}}, \\
\nabla \varphi=\nabla \boldsymbol{N}_{\varphi} \cdot \hat{\boldsymbol{\varphi}}, & \nabla \delta \varphi=\nabla \boldsymbol{N}_{\varphi} \cdot \delta \hat{\boldsymbol{\varphi}} .
\end{array}
$$

Let us introduce the displacement-strain operator $\boldsymbol{B}$ in Voigt-notation (denoted by $(\tilde{\cdot}))$ via

$$
\tilde{\varepsilon}=\boldsymbol{B} \cdot \hat{\boldsymbol{u}}, \quad \delta \tilde{\varepsilon}=\boldsymbol{B} \cdot \delta \hat{\boldsymbol{u}} .
$$

Inserting these approximations into our equations for the displacements (24) and the transportation function (25), we get

$$
\left[\begin{array}{c}
\int_{\Omega} \boldsymbol{B}^{T} \cdot \tilde{\boldsymbol{\sigma}} \mathrm{d} V-\int_{\Omega} \boldsymbol{N}_{\boldsymbol{u}} \cdot(\rho \boldsymbol{b}) \mathrm{d} V-\int_{\partial \Omega} \boldsymbol{N}_{\boldsymbol{u}} \cdot \boldsymbol{t} \mathrm{d} A \\
\int_{\Omega} c_{\varphi}\left(\nabla \boldsymbol{N}_{\varphi} \cdot \nabla \boldsymbol{N}_{\varphi} \cdot \hat{\boldsymbol{\varphi}}\right) \mathrm{d} V+\int_{\Omega} \beta_{\varphi}\left(\varphi+1-\left\|\boldsymbol{\lambda}_{0}\right\|\right) \boldsymbol{N}_{\varphi} \mathrm{d} V
\end{array}\right]=\left[\begin{array}{l}
\boldsymbol{R}_{\boldsymbol{u}} \\
\boldsymbol{R}_{\varphi}
\end{array}\right]=\left[\begin{array}{l}
\mathbf{0} \\
\mathbf{0}
\end{array}\right]
$$

Since the equations (30) are non-linear, a Newton algorithm is used to find the solution

$$
\left[\begin{array}{c}
\boldsymbol{R}_{\boldsymbol{u}} \\
\boldsymbol{R}_{\varphi}
\end{array}\right]^{i+1}=\left[\begin{array}{l}
\boldsymbol{R}_{\boldsymbol{u}} \\
\boldsymbol{R}_{\varphi}
\end{array}\right]^{i}+\boldsymbol{J}^{i} \cdot\left[\begin{array}{l}
\Delta \hat{\boldsymbol{u}} \\
\Delta \hat{\varphi}
\end{array}\right]^{i+1}=\left[\begin{array}{l}
\mathbf{0} \\
\mathbf{0}
\end{array}\right],
$$

with

$$
\boldsymbol{J}^{i}=\left[\begin{array}{ll}
\frac{\partial \boldsymbol{R}_{u}}{\partial \hat{\boldsymbol{u}}} & \frac{\partial \boldsymbol{R}_{u}}{\partial \hat{\boldsymbol{\varphi}}} \\
\frac{\partial \boldsymbol{R}_{\varphi}}{\partial \hat{\boldsymbol{u}}} & \frac{\partial \boldsymbol{R}_{\varphi}}{\partial \hat{\boldsymbol{\varphi}}}
\end{array}\right]^{i} .
$$

The entries in the Jacobian matrix (32) can be derived as

$$
\begin{aligned}
\frac{\partial \boldsymbol{R}_{\boldsymbol{u}}}{\partial \hat{\boldsymbol{u}}} & =\int_{\Omega} \boldsymbol{B}^{T} \cdot \mathbb{C}_{\mathrm{eff}} \cdot \boldsymbol{B} \mathrm{d} V \\
\frac{\partial \boldsymbol{R}_{\boldsymbol{u}}}{\partial \hat{\boldsymbol{\varphi}}} & =\int_{\Omega} \boldsymbol{B}^{T} \cdot \frac{\partial \tilde{\boldsymbol{\sigma}}}{\partial \varphi} \otimes \boldsymbol{N}_{\varphi} \mathrm{d} V \\
\frac{\partial \boldsymbol{R}_{\varphi}}{\partial \hat{\boldsymbol{u}}} & =\int_{\Omega} \beta_{\varphi} \boldsymbol{N}_{\varphi} \otimes\left(-\frac{\partial\left\|\boldsymbol{\lambda}_{0}\right\|}{\partial \tilde{\boldsymbol{\varepsilon}}}\right) \cdot \boldsymbol{B} \mathrm{d} V \\
\frac{\partial \boldsymbol{R}_{\varphi}}{\partial \hat{\varphi}} & =\int_{\Omega} c_{\varphi}\left(\nabla \boldsymbol{N}_{\varphi}\right)^{T} \cdot \nabla \boldsymbol{N}_{\varphi} \mathrm{d} V+\int_{\Omega} \beta_{\varphi}\left(1-\frac{\partial\left\|\boldsymbol{\lambda}_{0}\right\|}{\partial \varphi}\right) \boldsymbol{N}_{\varphi} \otimes \boldsymbol{N}_{\varphi} \mathrm{d} V .
\end{aligned}
$$

\subsection{Finite-element implementation}

The micromechanical model for the evolution of the different phases is solved using a staggered Newton algorithm. The input for the model is the strain which can be found, as usual, by an application of the Gauß quadrature to calculate the integrals. So the model is evaluated at the Gauß points. The derivatives in (34) till (36) have to be found numerically for each Gauß point. The updated volume fractions affect the stiffness of the body. Therefore an iteration between the updated displacements and the resulting distribution of the phases has to be executed until the change between the last two iteration steps is sufficiently small. 


\section{Discussion}

\subsection{Numerical results}

As numerical example we present a specimen made of CuAlNi for which we use the material data from [15]. This specimen is modeled with one layer of full 3D elements (since the traction is equal to zero, we calculate a plain stress problem). As boundary conditions we have a zero displacement at the left-hand side of the specimen and a time-depending non-zero displacement at the right-hand side.

In Figure (2) the distribution of the austenite phase is shown. The initial transformation arises at the shoulders of the body. Nevertheless the main transition starts in the middle of the specimen. The transformation remains quite localized and only starts to expand significantly when the austenite has nearly vanished in this area. Since the displacement of the right-hand side is still increasing, the transformation is proceeding through the body in fronts running to the left and the right. After the front has passed through the geometrically homogeneous part of the specimen, the amount of austenite is reduced in the transformed area even more. An further extension of the transformed part does not take place in a significant way.

Figure (3) shows the force-displacement diagram for the nodes of the right-hand side of the body. During the evolution of the front we can observe a softening of the material. After the front has moved through the body completely, the force remains constant.
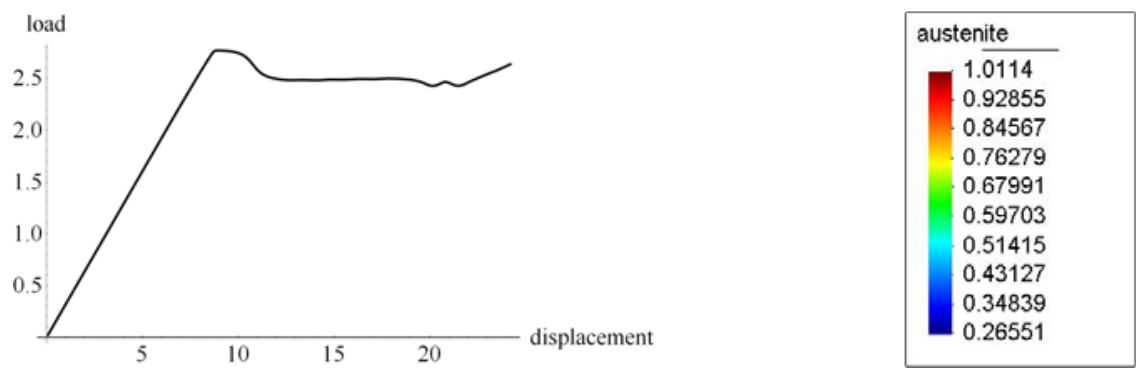

Fig. 3. Left: Force-displacement diagram of the nodes at the right-hand side of the specimen. Right: Legend for the distribution of the austenitic phase in figure (2).

\subsection{Conclusions}

Based on a micromechanical model we presented a new approach based on a modified dissipation functional. This leads to a field problem in which the informations are transported by a function $\varphi$ in order to get mesh-independent results. We suggested a solution strategy and provided appropriate numerical results which are in good agreement with experimental findings such as the occurrence of material inhomogeneities (called Lüders band), stress plateau and softening. In difference to the implementation of the common approach for the dissipation functional, the phase transformation takes place inhomogeneously. Particularly the front is well known from experimental findings. These bands are called Lüders bands. Interestingly the front in our numerical result is not perpendicular to the longitudinal axis of the specimen, as one may have expected. The occurring angle of not 90 is a subject of further investigation, due we expected to get this angle just by applying a small moment on the specimen which we did not. An analysis of the exact value of the angle with the measured angles from experiments is in our focus.

Due to the introduced field function the evolution of the internal variables is no longer coupled just by the strain field, but by the evolution of the internal variables themselves as well. That yields to smooth transitions of the internal variables throughout the body. Since "sharp" phase transitions in poly-crystalline experimental specimens come along with small areas (bands) where the lattice structures are changing continuously, we can observe this phenomenon in our numerical result as well. 

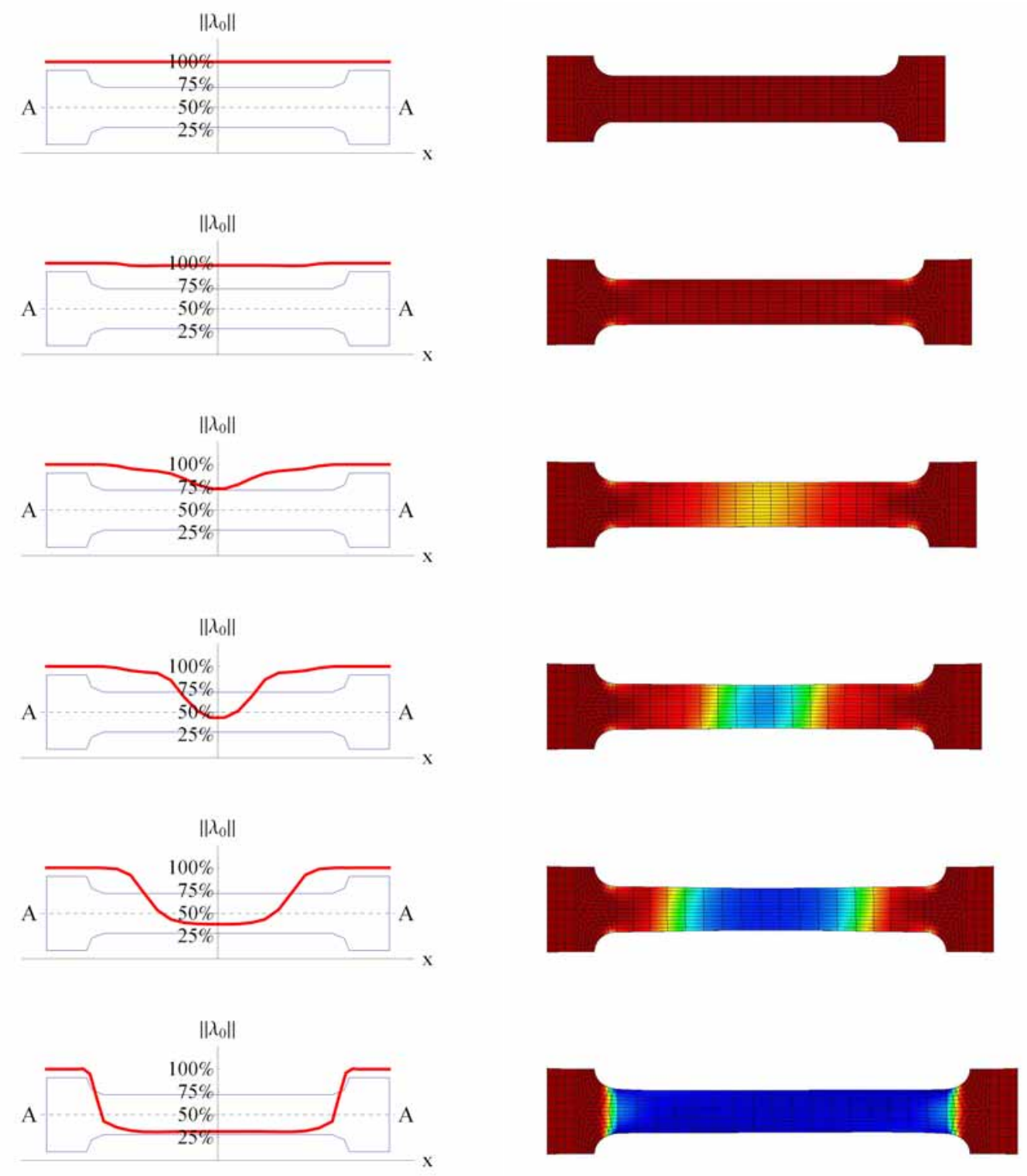

Fig. 2. Distribution of the austenitic phase at different load stages. Left: Contour line of the average amount of austenite at A-A. Right: Average amount of austenite within the specimen.

The decreasing stress at the beginning of the plateau is connected with the evolution of the front: As long as the front is evolving, the stress remains nearly constant. Softening is observed when the front is moving throughout the body. At that stage, the stress remains constant again, but on a lower level.

In result, the presented micromechanically well motivated algorithm is able to reflect experimental findings in a very good manner. Comparisons to experiments will enable us to fit the material parameters which will lead to a better understanding of the processes in a shapememory alloys. 


\section{References}

1. Ball, J. M. and James, R. D., Arch. Rat. Mech. Anal. 100, (1987) 13-52'

2. Bouvet, C., Calloch, S. and Lexcellent, C., Eur. J. Mech. A-Solids 23, (2004) 37-61

3. Dimitrijevic, B.J. and Hackl K., Technische Mechanik, 28, (2008) 43-52

4. Govindjee, S., Mielke, A., Hall, G. J., J. Mech. Phys. Solids 51, (2003) 763+

5. Govindjee, S., Hackl, K., Heinen, R., Continuum Mech. Thermodyn. 18, (2007) 443-453

6. Hackl, K. and Fischer, F. D., Proc. R. Soc. A 464, (2008) 117-132

7. Hackl, K. and Heinen, R., Continuum Mech. Thermodyn. 19, (2008) 499

8. Heinen, R. and Hackl, K., Comput. Meth. Appl. Mech. Eng. 196, (2007) 2401-2412

9. Helm, D. and Haupt, P., Int. J. Solids Struct. 40, (2003) 827-849

10. Kohn, R., Continuum Mech. Thermodyn. 3, (1991) 193-236

11. Mehrabadi, M.M. and Cowin, S.C., Q. J. Mech. Appl. Math. 100, (1990) 15-41

12. Mielke, A., Cont. Mech. Thermodyn. 15, (2003) 351-382

13. Ortiz, M. and Repetto, E.A., J. Mech. Phys. Solids 47, (1999) 397-462

14. Pan, H., Thamburaja, P. and Chau, F. S., Int. J. Plasticity 23, (2007) 711-732

15. Sedlák, P. et al, Acta Materialia 53, (2005) 3643-3661

16. Stupkiewicz, S. and Petryk, H., J. Mech. Phys. Solids 50, (2002) 2303-2331

17. Thamburaja, P., J. Mech. Phys. Solids 53, (2005) 825-856

18. Truskinovsky, L., Continuum Mech. Thermodyn. 6, (1994) 185-208 